\title{
Community-Acquired Pneumonia: A Practical Approach to Management for the Hospitalist
}

\author{
Bradley A. Sharpe, мо ${ }^{1}$ \\ Scott A. Flanders, $\mathrm{MD}^{2}$ \\ ${ }^{1}$ UCSF Department of Medicine, San Francisco, \\ California \\ ${ }^{2}$ University of Michigan, Ann Arbor, Michigan
}

\begin{abstract}
Community-acquired pneumonia (CAP) is common, and inpatient physicians should be familiar with the most current evidence about and guidelines for CAP management. Our conclusions and recommendations include: Streptococcus pneumoniae is the most common identified cause of CAP requiring hospitalization, whereas Legionella pneumophila is a common cause of severe CAP. The chest radiograph remains an essential initial test in the diagnosis of CAP and should be supplemented by blood cultures sampled prior to antibiotic therapy and sputum for gram stain and culture if a high-quality specimen can be rapidly processed. Once the diagnosis is made, the Pneumonia Severity Index (PSI) should be used to optimize the location of treatment and to provide prognostic information. Absent other mitigating factors, patients in PSI risk classes I, II, and III can safely be treated as outpatients. Hospitalized patients with CAP should be treated promptly with empiric antibiotics. Nonsevere pneumonia should be treated with a parenteral $\beta$-lactam plus either doxycycline or a macrolide. Patients admitted to the intensive care unit should be treated with a $\beta$-lactam plus either a macrolide or a fluoroquinolone as well as be evaluated for pseudomonal risk factors. Most patients with nonsevere CAP reach clinical stability in 2-3 days and should be considered for a switch to oral therapy and discharge shortly thereafter. Patients should receive pneumococcal vaccination, influenza vaccination, and tobacco cessation counseling prior to discharge if eligible. Multiple quality indicators are measured and publicly reported in the management of CAP, which provides hospitals with an opportunity to improve care processes and patient outcomes. Journal of Hospital Medicine 2006;1:177-190. (C) 2006 Society of Hospital Medicine.
\end{abstract}

KEYWORDS: community-acquired pneumonia, Streptococcus pneumonia, guidelines.

"Pneumonia may well be called the friend of the aged. Taken off by it in an acute, short, not often painful illness, the old man escapes those 'cold gradations of decay' so distressing of himself and to his friends."

—William Osler, MD, 1898

Community-acquired pneumonia (CAP) is commonly defined $\mathcal{U}_{\text {as }}$ an infection of the pulmonary parenchyma that is associated with at least some symptoms and signs of acute infection, accompanied by the presence of an acute infiltrate on chest radiograph, in a patient not hospitalized or residing in a long-termcare facility for 14 days prior to the onset of symptoms. ${ }^{1}$ CAP continues to be a common and serious illness, causing substantial morbidity and mortality in the adult population. There are an estimated 5-6 million cases a year in the United States, with greater than 1 million hospitalizations. Community-acquired pneumonia is one of the most common admitting diagnoses
Dr Flanders received speakers' honoraria from Elan, Pfizer, and Ortho-McNeil. 
TABLE 1

Most Common Pathogens in Community-Acquired Pneumonia by Site of Care ${ }^{5}$

\begin{tabular}{ll}
\hline Non-ICU inpatients & ICU inpatients (severe) \\
\hline S. pneumoniae & S. pneumoniae \\
M. pneumoniae & Legionella spp \\
C. pneumoniae & H. influenzae \\
H. influenzae & Gram-negative bacilli \\
Legionella spp & S. aureus \\
Aspiration & \\
Respiratory viruses & \\
\hline
\end{tabular}

among adults, and with a 30-day mortality between $10 \%$ and $14 \%$ for patients admitted to the hospital, it is the leading cause of infectious death in the United States. ${ }^{2}$ In elderly patients, hospitalization for CAP portends a poor long-term prognosis. In a Medicare database, the 1-year mortality for patients with CAP was nearly $40 \%$, compared to $29 \%$ in patients with other diagnoses. ${ }^{3}$ Community-acquired pneumonia is a model illness in hospital medicine-it is a common disease that allows for evidence-based and cost-effective management. In addition, many national organizations have proposed multiple quality indicators for community-acquired pneumonia, thus providing an opportunity for institutional quality improvement. This review article outlines the assessment and management of patients admitted to the hospital with communityacquired pneumonia.

\section{Etiology}

Although many pathogens can cause communityacquired pneumonia, the clinical syndromes and microbiology of CAP have traditionally been characterized as either "typical" or "atypical." The typical organisms include Streptococcus pneumoniae, Haemophilus influenzae, and Moraxella catarrhalis, and the atypical organisms include Chlamydia spp., Mycoplasma pneumoniae, Legionella spp., and viruses. This historical distinction has recently come into question. It is now clear that the presenting symptoms, signs, and basic laboratory findings (including the chest radiograph) cannot be reliably used to predict the etiologic pathogen or to distinguish typical from atypical organisms. ${ }^{4}$ Rather, the specific causative agent of CAP depends more on the degree of patient illness. Table 1 shows what prospective studies with comprehensive diagnostic
TABLE 2

Common Presenting Symptoms and Signs in Patients with Community-Acquired Pneumonia ${ }^{7,8}$

\begin{tabular}{ll}
\hline Symptoms & Signs (exam) \\
\hline Cough $90 \%$ & Fever $80 \%$ \\
Dyspnea $66 \%$ & Tachypnea 70\% \\
Sputum $66 \%$ & Tachycardia 50\% \\
Pleuritic chest pain 50\% & Focal lung exam $>90 \%$ \\
\hline
\end{tabular}

strategies determined to be the most common pathogens in patients hospitalized for CAP in ICU and non-ICU settings. ${ }^{5}$ Streptococcus pneumoniae remains the most common cause of CAP in hospitalized patients and is the most common cause of fatal pneumonia, whereas Legionella spp. is a common cause of severe CAP, more often found in patients requiring admission to the intensive care unit. Gram-negative bacilli can cause CAP in elderly patients and those recently treated with broad-spectrum antibiotics or with underlying lung disease. Notably, though, despite improved diagnostic testing, only one quarter of all admitted patients with CAP have the etiologic agent defined, and therefore empiric therapy should be directed broadly at the most likely organisms. ${ }^{6}$

\section{Clinical Presentation}

Patients admitted to the hospital with CAP typically present with a brief history of respiratory complaints, including cough (greater than $90 \%$ ), dyspnea (66\%), sputum production (66\%), and pleuritic chest pain (50\%); see Table $2 .{ }^{7,8}$ In $10 \%$ $30 \%$ of patients, nonrespiratory complaints predominate, including headache, myalgias, fatigue, and gastrointestinal symptoms. ${ }^{6}$ Elderly patients, an increasing percentage of hospitalized patients, are less likely to present with typical CAP symptoms (such as cough) and more likely to have altered mental status as a presenting symptom. ${ }^{9}$

On physical examination, patients with CAP usually have signs of fever (80\%), tachypnea (70\%), and tachycardia (50\%); see Table 2 . Most will have a focal lung exam $(>90 \%)$ with findings ranging from crackles to bronchial breath sounds. ${ }^{10}$ No exam finding is specific for the diagnosis of pneumonia, but the absence of fever, tachycardia, and tachypnea significantly reduces the probability of CAP in patients with suspected pneumonia. ${ }^{10}$ Furthermore, similar to the clinical history, the physi- 
cal examination of elderly patients with community-acquired pneumonia is not specific or sensitive for the diagnosis of CAP. For example, up to $40 \%$ of elderly patients subsequently determined to have CAP may not have fever. ${ }^{11}$

Leukocytosis is common in patients with CAP; however, its absence does not rule out disease. ${ }^{12} \mathrm{~A}$ number of guidelines recommend laboratory evaluation of electrolytes, urea nitrogen, creatinine, liver enzymes, and bilirubin, although these are used primarily for prognostication and are not specifically useful in the diagnosis of CAP.

\section{DIAGNOSIS}

\section{Differential Diagnosis}

Given the nonspecific nature of the symptoms and signs associated with CAP, there is no single clinical feature or combination of clinical features that adequately rules in or out the diagnosis of CAP. Consequently, the differential diagnosis to be considered in patients with suspected CAP is broad. Noninfectious diseases can often present with similar clinical syndromes; these include congestive heart failure, exacerbation of chronic obstructive pulmonary disease (COPD), asthma, pulmonary embolism, and hypersensitivity pneumonitis. These diseases can often be distinguished with a thorough history and physical examination.

In addition, other upper- and lower-airway infectious diseases can have similar nonspecific signs and symptoms. In particular, pneumonia must often be differentiated from acute bronchitis, which as a diagnosis accounts for up to $40 \%$ of patients evaluated for cough (versus $5 \%$ for pneumonia). ${ }^{10}$ Patients with acute bronchitis frequently do not present with high fevers or hypoxia and in general will not benefit from antibiotic therapy. ${ }^{13}$ Patients believed to have community-acquired pneumonia might also be suffering from other "pneumonia syndromes" including aspiration pneumonia, postobstructive pneumonia, and pneumonia in immunocompromised patients (eg, those with HIV, on steroids, receiving chemotherapy). Determining the correct diagnosis can have implications for therapy and prognosis.

\section{Diagnostic Studies}

The diagnosis of community-acquired pneumonia requires that a patient have both signs and symptoms consistent with pulmonary infection and evidence of a new radiographic infiltrate. Therefore, most guidelines recommend that all patients with a

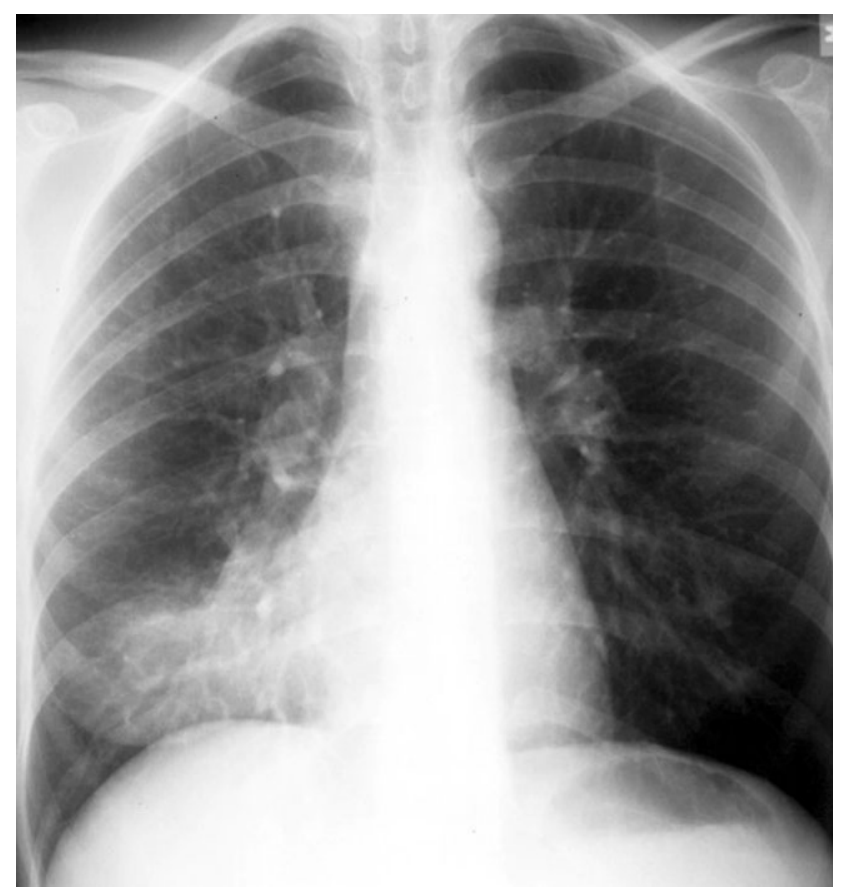

FIGURE 1. Chest radiograph of right middle lobe infiltrate in a patient with CAP.

possible diagnosis of CAP be evaluated with chest radiography. ${ }^{1,14,15}$

The specific radiographic findings in community-acquired pneumonia range from lobar consolidation to hazy focal infiltrate to diffuse bilateral interstitial opacities (see Figure 1). Although chest radiography has traditionally been considered the gold standard for the diagnosis of CAP, its exact performance characteristics are unknown, and it is clearly not $100 \%$ sensitive or $100 \%$ specific. The utility of the chest radiograph can be limited by patient body habitus, underlying lung disease, or dehydration. Computed tomography (CT) scanning, although not recommended for routine use, can identify pulmonary consolidation in up to $30 \%$ of patients with a normal or equivocal chest radiograph in whom pneumonia is suspected and can also identify complications of pneumonia including an empyema or pulmonary abscess. ${ }^{16}$

Limitations in the performance of the chest radiograph have resulted in an interest in the diagnostic performance of serologic markers of infection such as C-reactive protein (CRP), procalcitonin, and soluble triggering receptor expressed on myeloid cells (s-TREM). ${ }^{17-19}$ Preliminary evidence suggests these inflammatory markers may ultimately prove useful in differentiating infectious 
TABLE 3

Independent Predictors of Bacteremia in Patients with CommunityAcquired Pneumonia ${ }^{24}$

Comorbidities
Liver disease
Vital signs
Systolic blood pressure $<90 \mathrm{~mm} \mathrm{Hg}$
Temperature $<35^{\circ} \mathrm{C}$ or $\geq 40^{\circ} \mathrm{C}$
Pulse $\geq 125$ beats $/ \mathrm{min}$
Laboratory and radiographic data
Blood urea nitrogen (BUN) $\geq 30 \mathrm{mg} / \mathrm{dL}$
Sodium $<130 \mathrm{mmol} / \mathrm{L}$
White blood cells $<5000 / \mathrm{mm}^{3}$ or $>20,000 / \mathrm{mm}^{3}$
Prior use of antibiotics (negative predictor)

from noninfectious pulmonary processes, but regular use of these new tests cannot currently be recommended.

Most expert guidelines state that 2 sets of blood cultures should be taken and analyzed prior to antibiotic administration in all patients admitted to the hospital with suspected community-acquired pneumonia. ${ }^{1,14,15}$ Isolation of bacteria from blood cultures in CAP is a very specific way to identify a causative organism in order to subsequently narrow therapy and also identifies a high-risk group of patients because bacteremia is associated with increased mortality. Obtaining blood cultures within 24 hours of admission has been associated with $10 \%$ lower odds of 30-day mortality in patients with $\mathrm{CAP},{ }^{20}$ and as a result, drawing blood cultures prior to antibiotic administration is a national quality indicator for CAP.

There are, however, a number of problems with the routine acquisition of blood cultures in all patients admitted with CAP. Practically, the cultures can be difficult to obtain, can potentially delay the initiation of antibiotics, and are often contaminated, which has been shown to increase both cost and length of stay. ${ }^{21,22}$ The yield is generally low: the true-positive bacteremia rate for admitted patients with CAP ranges from $6 \%$ to $9 \%$, and the culture results rarely change management or outcomes. $^{23,24}$ Given these limitations, many have argued that blood cultures should be obtained with a more "targeted" approach. A recent study used a Medicare database to create a decision-support tool to help maximize the value of blood cultures in CAP. ${ }^{25}$ The predictors of a positive blood culture are shown in Table 3. Not obtaining cultures on patients who had received prior antibiotics or had no risk factors resulted in about $40 \%$ fewer overall cul- tures while identifying approximately $90 \%$ of bacteremias. In their guidelines, the British Thoracic Society (BTS) advocates a similar strategy, recommending blood cultures be omitted in nonsevere pneumonia and in patients without comorbidities. ${ }^{15,26}$ Although recommendations vary for nonsevere CAP in hospitalized patients, all professional society guidelines agree that blood cultures should be obtained in critically ill patients, and if cultures are obtained, they should be drawn prior to antibiotics. ${ }^{1,14,15,26}$

Substantial controversy surrounds the utility of routine sputum gram stains and cultures for patients admitted to the hospital with CAP. The Infectious Disease Society of America (IDSA) and the British Thoracic Society (BTS) both recommend that all patients admitted to the hospital with community-acquired pneumonia should have a gram stain and culture of expectorated sputum. ${ }^{1,15,26}$ Both organizations argue sputum collection is a simple and inexpensive procedure that can potentially identify pathogenic organisms and can affect both initial and long-term antibiotic therapy. Most notably, they highlight gram stain specificity of greater than $80 \%$ for pneumococcal pneumonia. Conversely, the American Thoracic Society (ATS) argues that sputum gram stains and cultures generally have low sensitivity, specificity, and positive predictive value. ${ }^{14}$ Furthermore, they argue the utility of sputum testing is also limited practically; in one study $30 \%$ of patients could not produce an adequate sputum specimen and up to $30 \%$ had received prior antibiotic therapy, substantially reducing the yield. ${ }^{27}$ In another study, good-quality sputum with a predominant morphotype could be obtained in only $14 \%$ of patients admitted with CAP.$^{28}$ However, targeting sputum analysis to patients who have not received prior antibiotics and are able to produce an adequate sample improved the yield significantly. ${ }^{29}$ In addition, with increasing rates of antibiotic resistance among common community isolates (ie, S. Pneumoniae) and the increasing prevalence of infecting organisms not targeted by routine empiric therapy (methicillin-resistant Staphylococcus Aureus [MRSA]), isolation of potential causative pathogens is increasingly important. We believe that severely ill patients with CAP (such as patients admitted to the ICU), as well as patients with identifiable risk factors for uncommon or drug-resistant pathogens (eg, Pseudomonas aeruginosa, enteric gram-negative rods, MRSA, etc.) should have sputum sent for gram stain and cul- 
ture. Ideally, sputum obtained for gram stain and culture should be:

1. Prior to antibiotic therapy,

2. A deep-cough, expectorated specimen,

3. A purulent specimen ( $>25$ polymorphonucleacytes and less than 10 squamous cells per highpowered field), and

4. Rapidly transported to the laboratory.

Subsequent gram stain and culture results should be interpreted in the specific clinical context and antibiotic choices targeted appropriately.

\section{Alternative Diagnostic Tests}

In recent years, there has been growth in additional diagnostic tests targeting specific organisms. The pneumococcal urinary antigen assay is a relatively sensitive (50\%-80\%) and highly specific $(\sim 90 \%)$ test for the detection of pneumococcal pneumonia, when compared with conventional diagnostic methods. ${ }^{27}$ The test is simple, convenient, rapid ( $15 \mathrm{~min}$ ), and, with its high specificity, may allow for more focused antimicrobial therapy early in management. Current limitations include the possibility of false-positive tests in patients colonized with $S$. pneumoniae or infected with other streptococcal species, as well as the inability to determine antibiotic sensitivity from positive tests. Updated IDSA and BTS guidelines state pneumococcal urinary antigen testing "is an acceptable" adjunct to other diagnostic tests, but blood and sputum analyses should still be performed. ${ }^{26,27}$ For patients with suspected Legionella pneumonia (primarily critically ill and immunocompromised patients or in association with regional outbreaks), the urinary Legionella antigen assay is the test of choice, which detects $80 \%-95 \%$ of community-acquired cases of Legionnaires' disease with a specificity of $90 \% .^{27}$

During the winter months (typically from October to March), rapid antigen testing for influenza is generally recommended for patients with signs or symptoms consistent with influenza. ${ }^{27}$ The sensitivity of these tests is approximately $50 \%-70 \%$, so negative test results do not exclude the diagnosis, but results can be important epidemiologically and therapeutically (differentiating influenza A and B strains) ${ }^{27}$ Diagnostic tests targeting other common CAP pathogens, such as serologic tests for Mycoplasma pneumoniae or Chlamydia spp, should not be routinely performed. Testing for less common causative pathogens such as Mycobacterium tuber- culosis should only be employed in the appropriate clinical setting.

\section{ADMISSION DECISION}

Once the diagnosis of CAP has been made, the initial site where treatment will occur, whether the hospital or the home, must be determined. The decision to hospitalize should be based on 3 factors: 1) evaluation of the safety of home treatment, 2) calculation of the Pneumonia Severity Index (PSI), and 3) clinical judgment of the physician. ${ }^{27}$ The PSI, or PORT (Pneumonia Outcomes Research Team) score, is a validated prediction rule that quantifies mortality and allows for risk stratification of patients with community-acquired pneumonia. ${ }^{2}$ The PSI combines clinical history, physical examination, and laboratory data at the time of admission to divide patients into 5 risk classes and to estimate 30 -day mortality (Figure 2), which ranges from $0.1 \%$ of patients in risk class I to $27.0 \%$ in risk class $V^{2}{ }^{2}$

On the basis of the estimated prognosis and in the absence of concerns about home safety or comorbidities, patients in risk classes I, II, and III should be managed at home. Many prospective trials have shown that implementation of PSI significantly increases the number of low-risk patients managed outside the hospital, with no differences in quality of life, complications, readmissions, or short-term mortality. ${ }^{30,31}$ Most recently, a trial randomizing patients in risk classes II and III to treatment in the hospital or at home found no significant differences in clinical outcomes but did find that patients were more satisfied with care at home. ${ }^{32}$ Because the number of patients with CAP being treated at home is increasing, the American College of Chest Physicians recently published a consensus statement on the management of community-acquired pneumonia in the home. ${ }^{33}$ All national guidelines for the management of community-acquired pneumonia recommend using the PSI to help determine the initial location of treatment, with the caveat that using the prediction rule should never supersede clinical judgment in the decision about whether to admit. ${ }^{1,14,15,26,27}$ A practical decision tree for the use of the PSI is shown in Figure 3.

There are no reliable prediction rules for deciding on whether admission to the intensive care unit is necessary. Hemodynamic instability requiring resuscitation and monitoring or respiratory failure requiring ventilatory support are clear indications 


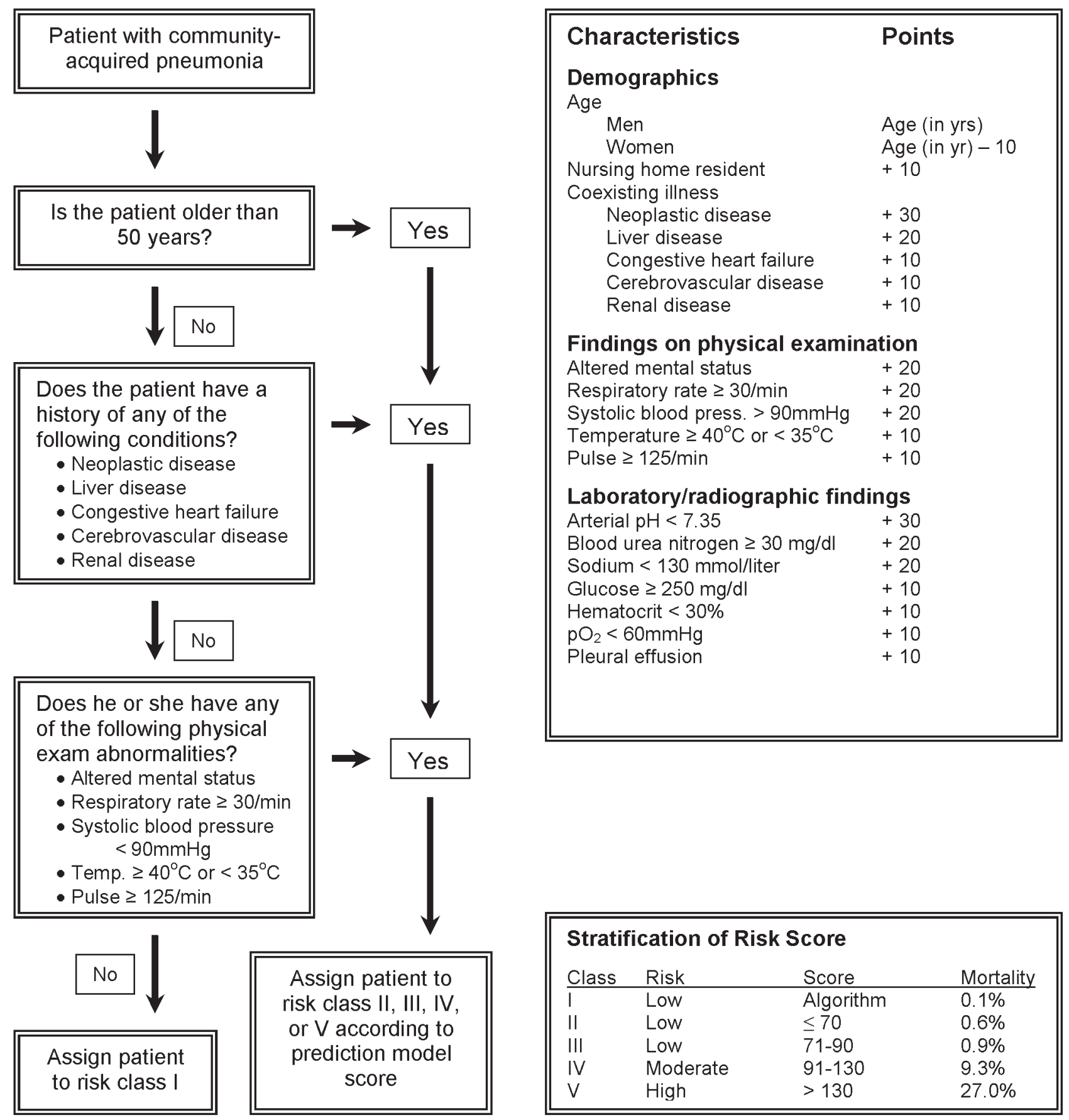

FIGURE 2. Pneumonia Severity Index (PSI), a validated prediction tool to estimate prognosis and aid in admission decisions for patients with community-acquired pneumonia (CAP). The score was calculated by adding the patient's age (in years for men or in years -10 for women) plus the points for each applicable characteristic. (Adapted from Halm EA, Teirstein AS. Management of community-acquired pneumonia. N Engl J Med. 2002;347(25):2039-2045 with permission. Copyright $\odot$ 2002. Massachusetts Medical Society. All rights reserved.)

for ICU admission. Additional variables such as tachypnea (respiratory rate $\geq 30$ ), altered mental status, multilobar disease, and azotemia are asso- ciated with severe CAP and should prompt consideration of ICU admission, especially when 2 or more variables coexist. ${ }^{14}$ 


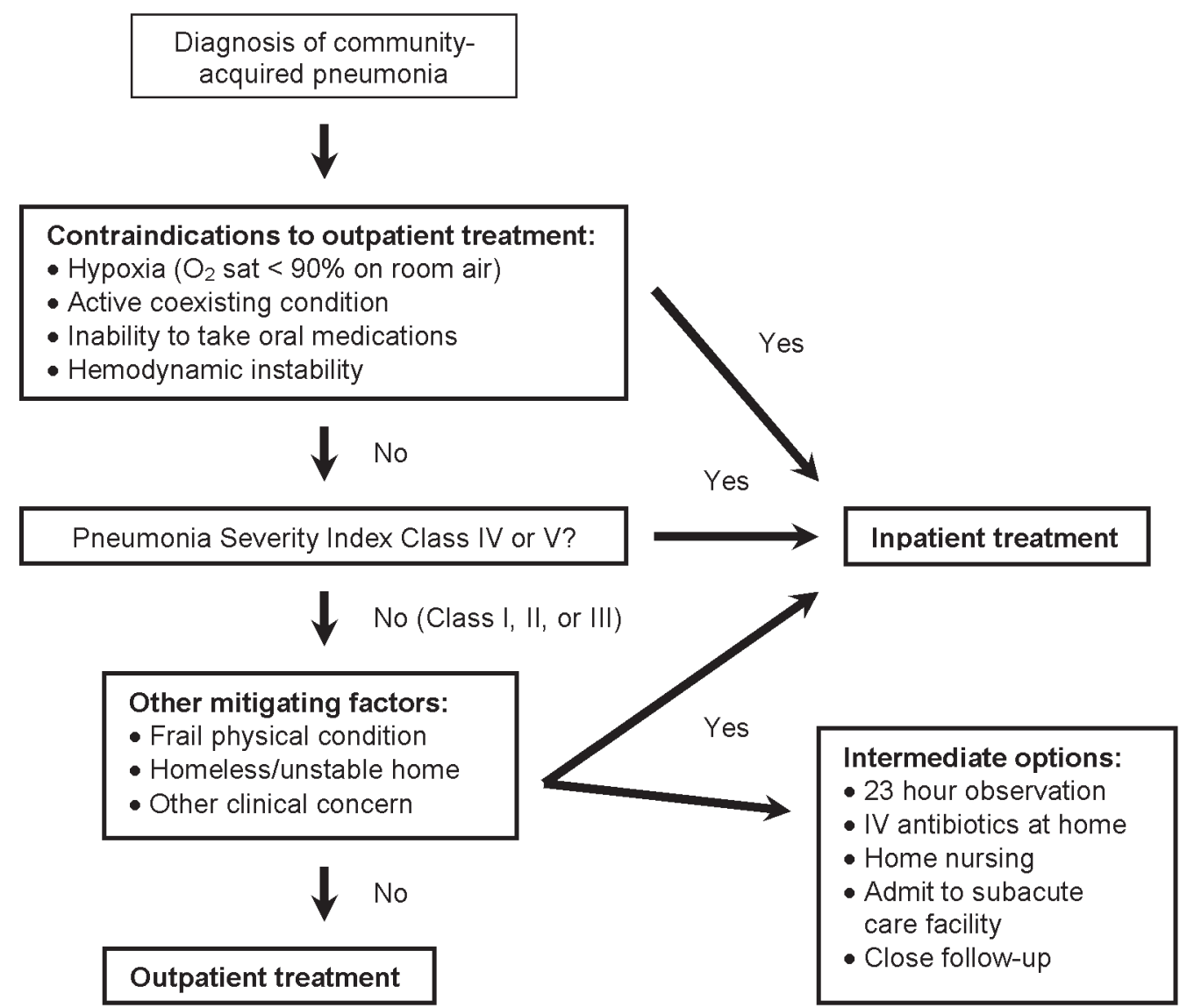

FIGURE 3. A practical decision-making tree using the PSI to aid in making the decision to admit. (Adapted from Halm EA, Teirstein AS. Management of community-acquired pneumonia. N Engl J Med. 2002;347(25):2039-2045 with permission. Copyright $\odot$ Massachusetts Medical Society. All rights reserved.)

\section{TREATMENT \\ Initial Treatment}

Once the admission decision is made and the initial diagnostic tests are completed (including blood and sputum cultures), patients with presumed community-acquired pneumonia should receive necessary supportive care $\left(\mathrm{O}_{2}\right.$, intravenous fluids, etc.) and prompt antimicrobial therapy. Antibiotics should be administered within 4 hours of arrival to patients with suspected CAP, as such prompt administration may be associated with shorter in-hospital stays and decreased 30-day mortality. ${ }^{34,35}$ Regulatory organizations such as the Joint Commission on Accreditation of Healthcare Organizations (JCAHO) and the Center for Medicare Services (CMS) have made delivery of antibiotics in less than 4 hours a hospital quality measure.

Despite diagnostic testing, the specific etiologic agent causing the pneumonia of a patient remains unknown in up to $75 \%$ of those admitted to the hospital. ${ }^{14}$ Most expert guidelines therefore recommend broad-spectrum empiric therapy targeting both the "typical" and the "atypical" organisms that commonly cause CAP (Table 1).

Recommendations for empiric antibiotics are driven by 2 key factors: antibiotic resistance by $S$. pneumoniae and the results of studies of CAP treatment outcomes. Historically, patients with suspected community-acquired pneumonia were treated with penicillin with generally good outcomes. Recently, the rate of S. pneumoniae isolates resistant to penicillin has risen dramatically in the United States, ranging between $20 \%$ and $30 \%$, with high-level resistance (MIC $\geq 4 \mathrm{mg} / \mathrm{L}$ ) as high as $5.7 \% .^{36,37}$ Concurrently, the rates of resistance of $S$. pneumoniae to many other antibiotics commonly used to treat CAP have also risen. ${ }^{37}$ Despite increasing resistance overall, most U.S. pneumococcal isolates have low resistance to third-generation cephalosporins and fluoroquinolones with enhanced 
activity against $S$. pneumoniae..$^{36-38}$ In addition, despite increasing resistance by pneumococcal isolates to penicillin, several observational studies have shown that regardless of initial therapy, resistance to penicillin as well as third-generation cephalosporins is not associated with higher mortality or worse outcomes when controlled for other risk factors for drug resistance. ${ }^{39,40}$ An exception to that rule is pneumococcal isolates that are very highly resistant to $\mathrm{PCN}$ (MIC $\geq 4 \mathrm{mg} / \mathrm{dL}$ ). At least one study has shown that patients with such isolates may be at higher risk for adverse outcomes and should probably not be treated with penicillins. ${ }^{1,14,15,41}$ However, nationally, fewer than $6 \%$ of pneumococcal isolates have this level of resistance. $^{37}$

The rationale for empiric broad-spectrum coverage against both typical and atypical organisms has arisen from many retrospective and observational studies that have suggested that there is clinical benefit and improved outcomes with such regimens. One large retrospective study showed that in elderly patients with CAP, fluoroquinolone monotherapy was associated with lower 30-day mortality when compared to monotherapy with a third-generation cephalosporin. ${ }^{34}$ Adding an extended-spectrum macrolide (eg, azithromycin) to an extendedspectrum $\beta$-lactam (eg, ceftriaxone) in the treatment of patients hospitalized with nonsevere CAP also appears to be associated with improved outcomes. Adding a macrolide has resulted in shorter lengths of stay (LOS), less treatment failure, and lower mortality. ${ }^{34,42-44}$ Similarly, according to unpublished observations, adding doxycycline to a $\beta$-lactam as initial therapy was associated with a benefit of decreased mortality. ${ }^{45}$ The presumed etiology of the benefit has been the addition of specific coverage of atypical organisms, such as Mycoplasma pneumoniae and Chlamydia pneumoniae, which are common causes of CAP (Table 1). Others have proposed that the benefit of therapy with macrolides may be derived from the inherent antiinflammatory properties of macrolides. ${ }^{46}$ Because research has shown a benefit of dual versus monotherapy across a spectrum of antibiotics, others have proposed the benefit is simply a result of receiving "double" antibiotic coverage. In particular, 2 studies found a benefit of reduced mortality from combination therapy over monotherapy in bacteremic pneumococcal pneumonia. ${ }^{47,48}$

Yet the accumulated evidence for adding coverage of atypical organisms has been only retro- spective and observational. Because of this, the recommendation to routinely add antibiotics active against atypical organisms has been questioned by some. Two recent meta-analyses and a systematic review examined all the available data on the need for atypical coverage in the treatment of patients with community-acquired pneumonia. ${ }^{49-51}$ Surprisingly, none showed a benefit in clinical efficacy or survival in patients treated with agents active against both atypical and typical organisms when compared to regimens with only typical coverage. In subset analyses, there was a benefit to providing empiric atypical coverage in patients subsequently shown to have Legionella spp. as a causative pathogen. However, this organism was uncommon in all 3 studies. Unfortunately, most studies included in the meta-analyses compared fluoroquinolone or macrolide monotherapy with third-generation cephalosporin monotherapy. There have been no high-quality randomized, controlled trials of the treatment of hospitalized patients with CAP assessing combination therapy covering both typical and atypical organisms with monotherapy targeting typical organisms alone. High-quality trials are warranted.

Despite the recent articles questioning the importance of atypical coverage, citing the substantial retrospective data and the general inability to identify causative organisms in most cases of CAP, adding a second agent with atypical coverage to a $\beta$-lactam currently appears to be the most efficacious empiric treatment for CAP. Nearly all expert guidelines for the management of community-acquired pneumonia recommend this empiric approach. ${ }^{1,14,27}$

Table 4 displays our recommendations for the treatment of community-acquired pneumonia requiring hospitalization. Before implementation of these guidelines, hospitalists should consult with their infectious disease experts and consider local resistance patterns. In general, a typical adult patient with non-severe CAP without additional risk factors should receive a parenteral extended-spectrum $\beta$-lactam plus either doxycycline or an advanced macrolide (see Table 4). Extended-spectrum $\beta$-lactams include cefotaxime, ceftriaxone, ampicillin-sulbactam, and ertapenem. A respiratory fluoroquinolone as a single agent can be used for non-ICU patients with CAP, but some agencies, including the Centers for Disease Control (CDC), discourage routine use of these agents in all patients secondary to concerns about cost and in- 
TABLE 4

Suggested Initial Empiric Antimicrobial Therapy in Immunocompetent Patients with Suspected Community-Acquired Pneumonia

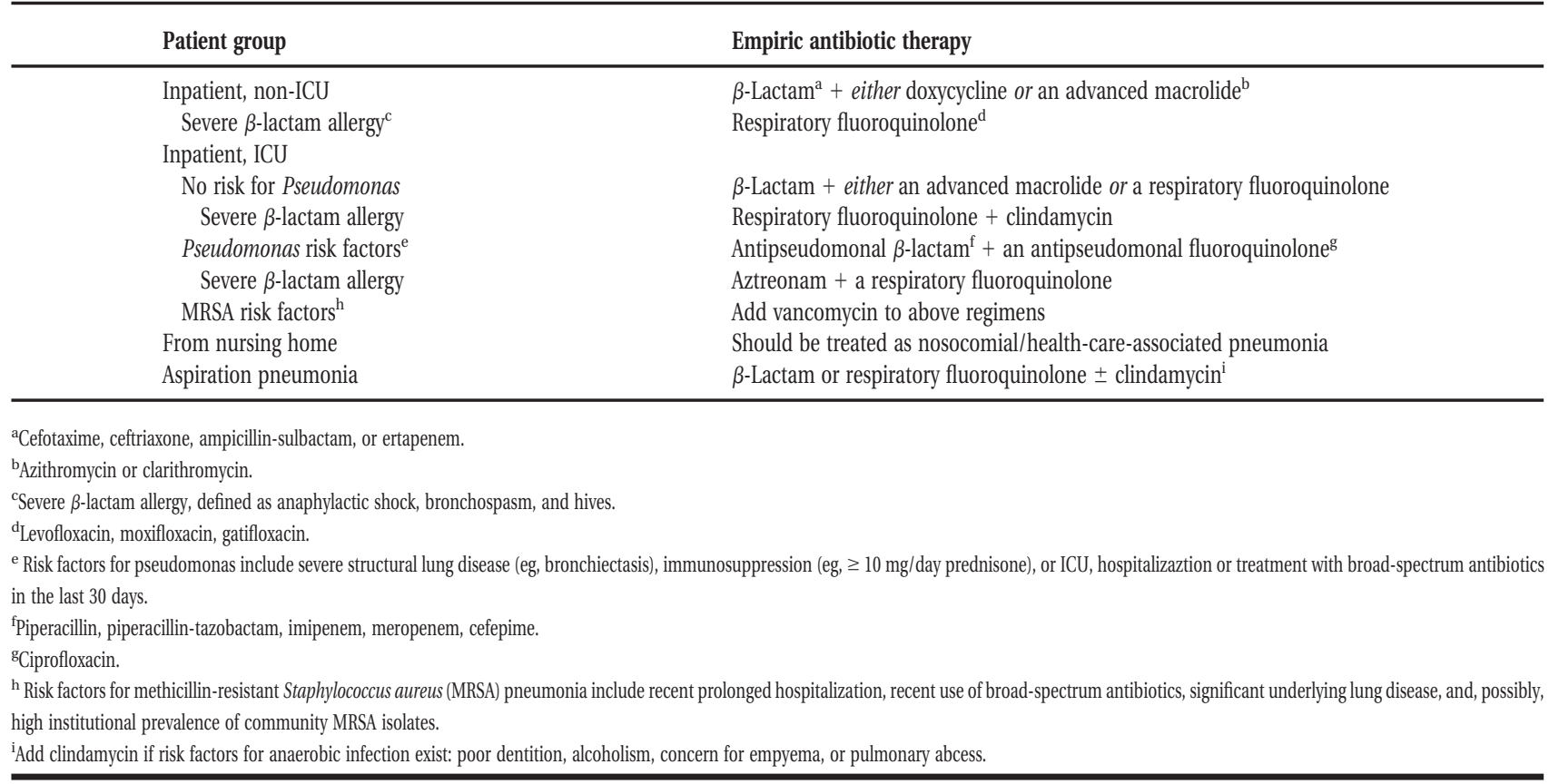

creasing gram-negative rod fluoroquinolone resistance. ${ }^{52,53}$

Patients hospitalized with severe CAP who require ICU-level care are at increased risk of Legionella spp. and drug-resistant S. pneumoniae, which must be reflected in their initial antibiotic therapy. ${ }^{5}$ Patients with severe pneumonia should receive an intravenous extended-spectrum $\beta$-lactam plus either an intravenous macrolide or an intravenous respiratory fluoroquinolone.

All patients with severe CAP who are admitted to the intensive care unit should be routinely screened for risk factors for Pseudomonas aeruginosa. The known risk factors for pseudomonal infection are: bronchiectasis, immunosuppression including more than $10 \mathrm{mg} /$ day of prednisone, malnutrition, and treatment with broad-spectrum antibiotics in the last month. ${ }^{14}$ Those at risk for Pseudomonas aeruginosa or other resistant gramnegative rod infection should be treated with an antipseudomonal $\beta$-lactam plus an antipseudomonal fluoroquinolone. Many patients with severe CAP have risk factors for MRSA infection including recent prolonged hospitalization, recent use of broad-spectrum antibiotics, and significant underlying lung disease, which should be considered in choosing initial antibiotic therapy. ${ }^{54}$ In addition, there have been reports of patients without underlying risk factors presenting with severe community-acquired MRSA pneumonia. Many of these patients were younger and the MRSA pneumonia was associated with a necrotizing or cavitary disease requiring prolonged ICU stays. ${ }^{55-58}$ In such cases or if an institution's rate of methicillin resistance in $S$. aureus community isolates is high $(>15 \%-20 \%)$, it may be appropriate to add initial empiric MRSA coverage for patients admitted to the ICU with CAP. ${ }^{55}$

Some patients will have unique risk factors and clinical presentations, which may require modification of these empiric recommendations. Several studies found $5 \%-15 \%$ of cases of community-acquired pneumonia to be aspiration pneumonia. ${ }^{57}$ Risk factors for aspiration events include, among others, dysphagia, history of stroke, altered level of consciousness, poor dentition, and tube feeding. Aspiration pneumonia traditionally was believed to be secondary to oral anaerobes, but recent research suggests gram-positive cocci and gram-negative rods are the predominant organisms. ${ }^{58}$ Antibiotic therapy in patients with clear aspiration pneumonia should be directed at these microbes with an extended-spectrum $\beta$-lactam (eg, ceftriaxone) or a respiratory fluoroquinolone (eg, levofloxacin or 
TABLE 5

Criteria to Identify Clinically Stable Patients for Potential Switch to Oral Antibiotic Therapy ${ }^{27}$

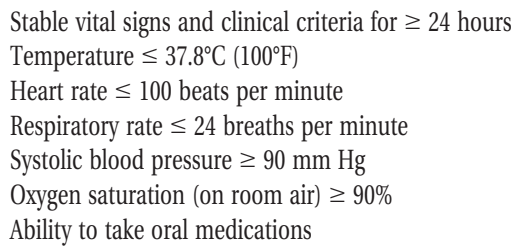

moxifloxacin). Anaerobic bacterial coverage can be added in patients with severe periodontal disease, alcoholism, concern for empyema, or evidence of aspiration with pulmonary abscess. ${ }^{58}$

Patients residing in long-term care facilities are at high risk of contracting pneumonia. The microbiology of infections acquired in nursing facilities is similar to that in hospital-acquired cases. ${ }^{59,60}$ As a result, patients who develop pneumonia in institutional settings such as nursing homes should be treated with broad-spectrum antibiotics, including coverage for MRSA.

\section{Subsequent Treatment}

Initial empiric antibiotic treatment should be modified based on the results of diagnostic testing. Although the specific etiologic agent is determined in only $25 \%$ of cases of CAP, ${ }^{35}$ when an organism is isolated, antibiotic coverage should be narrowed to cover that particular organism with an antibiotic with adequate lung penetration. Evidence suggests clinicians often do not adjust or narrow antibiotics based on sensitivity results, potentially breeding resistant organisms. ${ }^{61}$

Patients hospitalized with CAP usually improve quickly if they receive early, appropriate antibiotic therapy and supportive care. Excluding patients with severe CAP requiring intensive care unit admission, most patients resolve their tachycardia, tachypnea, and fever by day 2 or $3 .^{62}$ Recent practice experience, evidence, and published guidelines ${ }^{14,27}$ all indicate that patients can safely be transitioned to oral antibiotic therapy earlier in their hospital course. Table 5 outlines criteria that can be used to identify patients who have had an adequate response to parenteral therapy and can be considered for a switch to oral antibiotics. If these criteria are met, patients have less than a $1 \%$ chance of clinical deterioration necessitating admission to an ICU or transitional care unit. ${ }^{62}$ When an etiologic organism is not identified, oral therapy should reflect a spectrum of coverage to that of the initial intravenous therapy. In some cases, this may require use of more than one oral agent. We have had success, however, transitioning non-ICU patients initially treated with intravenous ceftriaxone plus oral doxycycline, typically for 48-72 hours, to oral doxycycline monotherapy at discharge. ${ }^{45}$

There have been a limited number of highquality randomized trials examining the optimal duration of treatment for community-acquired pneumonia. Most practice guidelines recommend 7-10 days for patients with CAP requiring hospitalization, with 14 days for documented Mycoplasma pneumoniae or Chlamydia pneumoniae. One recent randomized trial of patients with mild to severe CAP showed a short course of high-dose levofloxacin (750 mg daily $\times 5$ days) was at least as effective as normal dosing $(500 \mathrm{mg}$ daily $\times 10$ days). ${ }^{63}$ Clinical experience with high-dose levofloxacin is limited, but this regimen can be considered because it may reduce costs and exposure to antibiotics. When diagnosed, Legionella is usually treated for 10-21 days, but 14 days is adequate with macrolides because of their long half-life. ${ }^{27}$ Patients with more virulent pathogens like Staphylococcus aureus or Pseudomonas aeruginosa or other suppurative complications should be treated for at least 14 days. ${ }^{1,14,15,27}$ In determining length of therapy, clinicians should use these durations of treatment as guides, and to individualize therapy, they should always consider patient age and frailty, comorbid conditions, severity of illness, and hospital course.

\section{Failure to Respond}

Although most patients hospitalized for CAP will improve rapidly and reach clinical stability in 2-3 days, some patients fail to respond. Some studies have estimated that failure to improve or clinical deterioration occurs in $5 \%-10 \%$ of patients in the first 2-3 days. ${ }^{64}$ The common reasons for clinical decline or nonresponse to treatment, highlighted in Table 6, are:

1. Incorrect diagnosis: Illnesses such as congestive heart failure, pulmonary embolism, neoplasms, and hypersensitivity pneumonitis can mimick CAP.

2. Inadequate antibiotic selection: The etiologic agent may be resistant to empiric antibiotic selections. Examples would include methicillin-resistant 
TABLE 6

Reasons for Clinical Deterioration or Nonresponse to Treatment in CAP

Incorrect diagnosis of CAP.

Inadequate or inappropriate antibiotic selection for CAP.

Unusual pathogen causing CAP.

Pulmonary or extrapulmonary complication of CAP.
Inadequate or poor host response.

TABLE 7

Criteria for Identifying Patients for Possible Discharge

Patients should:

Meet clinical criteria in Table V.

Be able to tolerate oral medications (no need to observe for 24 hours on oral therapy).

Have no evidence of active comorbid conditions (myocardial ischemia, pulmonary edema, etc.).

Have a normal mental status (or have returned to their baseline).

Have safe, appropriate outpatient follow-up.

Staphylococcus aureus (MRSA) or multiresistant gram-negative bacilli.

3. Unusual pathogen: CAP syndromes can be caused by myriad unusual organisms including Pneumocystis jirovecii, mycobacterium tuberculosis, endemic fungal infections (eg, coccidioidomycosis), and nocardiosis.

4. Complications of pneumonia: Specific complications of CAP include empyema, pulmonary abscess, extrapulmonary spread including meningitis or endocarditis, or other organ dysfunctions such as renal failure or myocardial infarction.

5. Inadequate host response: Despite appropriate antibiotic and supportive therapy, patients with CAP often fail to respond.

Progressive pneumonia despite appropriate therapy and empyema were the most common causes of failure to respond in the first 72 hours in a recent study. ${ }^{64}$ Risk factors for early failure were older age ( $>65$ years), Pneumonia Severity Index $>90$, Legionella pneumonia, gram-negative pneumonia, and initial antimicrobial therapy discordant with final culture and susceptibility results. The initial evaluation of the nonresponding patient should address these common causes and is likely to include additional imaging (CT), sampling of potential extrapulmonary infection (thoracentesis), and, in some cases, bronchoscopy.

\section{DISCHARGE/FOLLOW-UP PLANS}

Patients hospitalized for community-acquired pneumonia can be safely discharged when they have reached clinical stability, are able to tolerate oral medications, have no other active comorbid conditions, and have safe, close, appropriate outpatient follow-up (see Table 7). Clinical pathways employing these discharge criteria have been found to be safe and effective in reducing the length of stay for CAP. Most important, patients should have met most if not all of the vital sign and clinical criteria noted in Table 5 in the criteria for switching to oral therapy. Patients with 2 or more abnormal vital signs ("instabilities") within 24 hours prior to discharge are at high risk of readmission and mortality, but those with one or no abnormal vital signs generally have good outcomes. ${ }^{65}$ Absent other clinical factors or extenuating circumstances (persistent hypoxia, poor functional status, etc.), most patients with CAP should reach clinical stability by day 3 or 4, be considered for a switch to oral therapy, and, if stable, be discharged shortly thereafter.

When patients with CAP are discharged from the hospital, they should be counseled about the expected course of recovery. Most important, patients and families must be informed that many symptoms of CAP may persist well after hospitalization. In one study, up to $80 \%$ of patients reported persistent cough and fatigue 1 week after discharge, and up to $50 \%$ still had dyspnea and sputum production. In some, the cough can last for 4-6 weeks. ${ }^{8}$

All patients discharged after treatment of community-acquired pneumonia should have followup with their outpatient provider. The physician responsible for their inpatient care should communicate directly with the provider and outline the hospital course, the discharge medications, and the duration of antibiotic therapy. There is no specific time frame within which patients must be seen, but follow-up should be dictated by patient age, comorbidities, clinical stability at discharge, and degree of illness. The American Thoracic Society guidelines do recommend patients with a substantial smoking history who are hospitalized with CAP have a follow-up chest radiograph 4-6 weeks after discharge to establish a radiographic baseline and exclude the possibility of underlying malignancy. ${ }^{14}$ However, several studies have suggested that radiographic resolution may take 3 or more months in some patients, especially the elderly and those with multilobar disease. ${ }^{66}$ 


\section{PREVENTION}

Prevention of community-acquired pneumonia and pneumonic syndromes has traditionally relied on vaccination with the polysaccharide pneumococcal pneumonia vaccine and the seasonal influenza vaccine. The vaccine for $S$. pneumoniae used in adults is composed of the 23 serotypes that cause $85 \%-90 \%$ of the invasive pneumococcal infections in the United States. Although in randomized trials the vaccine has not consistently prevented community-acquired pneumonia or death in elderly patients or those with comorbidities, it likely prevents invasive pneumococcal infection. ${ }^{67}$ National guidelines and the CDC recommend the pneumococcal vaccine be given to all patients older than 65 years and those with chronic medical conditions. ${ }^{1,14,15}$

The seasonal influenza vaccine has clearly been shown to decrease influenza-related illness in elderly and high-risk patient populations. As well, in a meta-analysis and a large observational study of patients older than 65 years, vaccination against influenza prevented pneumonia, hospitalization, and death. ${ }^{6,69}$ Vaccination of health care workers may also confer a benefit to elderly patients of reduced mortality. The CDC recommends the influenza vaccine for all patients more than 50 years old, those with comorbidities, those at high risk for influenza, and health care workers in both inpatient and outpatient settings.

Pneumococcal and influenza vaccination have traditionally been relegated to the outpatient setting. National guidelines and the CDC recommend vaccination of all eligible hospitalized patients. Vaccination is safe and effective with almost any medical illness, and both vaccines can be given simultaneously at discharge. ${ }^{69}$ Both JCAHO and CMS have defined administration of the pneumococcal and influenza vaccines to patients hospitalized with CAP as a quality measure. Using "standing orders" is the most effective means of ensuring vaccination.

Some evidence suggests that tobacco smokers are at increased risk of invasive pneumococcal disease or pneumonia. ${ }^{70}$ Patients hospitalized (for all illnesses, but for CAP in particular) should be counseled about smoking cessation and offered pharmacotherapy and outpatient follow-up. And, finally, recent observational data suggests that use of acid suppressive therapy, including proton pump inhibitors and $\mathrm{H}-2$ receptor antagonists, may be associated with an increased risk of developing CAP. ${ }^{71}$ Patients using these agents who are admitted with
CAP should have their indications for treatment reviewed, especially when the pneumonia has been recurrent and there is no clear indication for continued use of acid suppressive therapy, in which case they should be discontinued in the hospital.

\section{CONCLUSIONS}

Community-acquired pneumonia remains a common cause for hospitalization of adult patients, with significant associated morbidity and mortality. Although there are multiple expert guidelines for the management of community-acquired pneumonia, further research is urgently needed. Clinicians need improved diagnostic tests that enable an earlier and more accurate diagnosis of CAP. In addition, the etiologic agent causing CAP is rarely discovered; improved microbiologic studies might enable antibiotic therapy to be targeted to the organisms responsible. High-quality randomized, controlled trials examining empiric antibiotic therapy in CAP are needed, especially related to the addition of agents covering atypical organisms. Last, the general management of patients hospitalized with CAP is marked by significant heterogeneity, and research and initiatives focusing on improving the quality and process of care of patients with CAP are needed.

Address for correspondence and reprint requests: Bradley A. Sharpe, MD, Assistant Clinical Professor and Assistant Chief of the Medical Service, UCSF Department of Medicine, 505 Parnassus Avenue, Box 0131, San Francisco, CA 94143; Fax: (415) 502-6235; E-mail: sharpeb@medicine.ucsf.edu

Received 21 December 2005; revision received 2 February 2006; accepted 3 February, 2006.

\section{REFERENCES}

1. Bartlett JG, Dowell SF, Mandell LA, File TM, Musher DM, Fine MJ. Guidelines from the Infectious Disease Society of America. Practice guidelines for the management of community-acquired pneumonia. Clin Infect Dis. 2000;31:347382.

2. Fine MJ, Auble TE, Yealy DM, et al. A prediction rule to identify low-risk patients with community-acquired pneumonia. N Engl J Med. 1997;336:243-250.

3. Kaplan V, Clermont G, Griffin MF, et al. Pneumonia: still the old man's friend? Arch Intern Med. 2003;163:317-323.

4. Fang GD, Fine M, Orloff J, et al. New and emerging etiologies for community-acquired pneumonia with implications for therapy. A prospective multicenter study of 359 cases. Medicine. 1990;69:307-316.

5. File TM. Community-acquired pneumonia. Lancet. 2003; 362:1991-2001. 
6. Fine MJ, Stone RA, Singer DE, et al. Processes and outcomes of care for patients with community-acquired pneumonia: results from the Pneumonia Patient Outcomes Research Team (PORT) cohort study. Arch Intern Med. 1999;159:970980.

7. Halm EA, Teirstein AS. Management of community-acquired pneumonia. $N$ Engl J Med. 2002;347:2039-2045.

8. Metlay JP, Fine MJ, Schulz R, et al. Measuring symptomatic and functional recovery in patients with community-acquired pneumonia. J Gen Intern Med. 1997;12:423-430.

9. Metlay JP, Schulz R, Li YH, et al. Influence of age on symptoms at presentation with patients with community-acquired pneumonia. Arch Intern Med. 1997;157:1453-1459.

10. Metlay JP, Kapoor WN, Fine MJ. Does this patient have community-acquired pneumonia? Diagnosing pneumonia by history and physical examination. JAMA. 1997;278:14401445.

11. Fernandez-Sabe N, Carratala J, Roson B, et al. Communityacquired pneumonia in very elderly patients: causative organisms, clinical characteristics, and outcomes. Medicine. 2003;82:159-169.

12. Metlay JP, Fine MJ. Testing strategies in the initial management of patients with community-acquired pneumonia. Ann Intern Med. 2003;138:109-118.

13. Gonzales R, Sande MA. Uncomplicated acute bronchitis. Ann Intern Med. 2000;133:981-991.

14. Niederman MS, Mandell LA, Anzqueto A, et al. American Thoracic Society: Guidelines for the management of community-acquired pneumonia: diagnosis, assessment of severity, antimicrobial therapy, and prevention. Am J Respir Crit Care Med. 2001;163:1730-1754.

15. Macfarlane J, Boswell T, Douglas G, et al. British Thoracic Society guidelines for the management of community acquired pneumonia in adults. Thorax. 2001;56:Suppl. 4, IV1IV64.

16. Syrjala H, Broas M, Suramo I, Ojala A, Lahde S. High-resolution computed tomography for the diagnosis of community-acquired pneumonia. Clin Infect Dis. 1998;27:358-363.

17. Flanders SA, Stein J, Shochat G, et al. Performance of a bedside C-reactive protein test in the diagnosis of community-acquired pneumonia in adults with acute cough. Am J Med. 2004;116:529-535.

18. Christ-Crain M, Jaccard-Stolz D, Bingisser R, et al. Effect of procalcitonin-guided treatment on antibiotic use and outcome in lower respiratory tract infections: cluster-randomised, single-blinded intervention trial. Lancet. 2004;363:600607.

19. Gibot S, Cravoisy A, Levy B, et al. Soluble triggering receptor expressed on myeloid cells and the diagnosis of pneumonia. N Engl J Med. 2004:350:451-458.

20. Meehan TP, Fine MJ, Krumholz HM, et al. Quality of care, process, and outcomes in elderly patients with pneumonia. JAMA. 1997;278:2080-2084.

21. Corbo J, Friedman B, Bijur P, Gallagher EJ. Limited usefulness of initial blood cultures in community acquired pneumonia. Emerg Med J. 2004;21:446-448.

22. Bates DW, Goldman L, Lee TH. Contaminant blood cultures and resource utilization. The true consequences of falsepositive results. JAMA. 1991;265:365-369.

23. Campbell SG, Marrie TJ, Anstey R, et al. The contribution of blood cultures to the clinical management of adult patients admitted to the hospital with community-acquired pneu- monia: a prospective observational study. Chest. 2003;123: 1142-1150.

24. Chalasani NP, Valdecanas MA, Gopal AK, et al. Clinical utility of blood cultures in adult patients with communityacquired pneumonia without defined underlying risks. Chest. 1995;108:932-936.

25. Metersky ML, Ma A, Bratzler DW, Houck PM. Predicting bacteremia in patients with community-acquired pneumonia. Am J Respir Crit Care Med. 2004;169:342-347.

26. British Thoracic Society. BTS Guidelines for the management of community acquired pneumonia in adults-2004 update. Available at: www.brit-thoracic.org/guidelines.

27. Mandell LA, Bartlett JG, Dowell SF, File TM, Musher DM, Whitney C. Guidelines from the Infectious Disease Society of America. Update of guidelines for the management of community-acquired pneumonia in immunocompetent adults. Clin Infect Dis. 2003;37:1405-1433.

28. Garcia-Vazquez E, Marcos MA, Mensa J, et al. Assessment of the usefulness of sputum culture for diagnosis of community-acquired pneumonia using the PORT predictive scoring system. Arch Intern Med. 2004;164:1807-1811.

29. Musher DM, Montoya R, Wanahita A. Diagnostic value of microscopic examination of gram-stained sputum and sputum cultures in patients with bacteremic pneumococcal pneumonia. Clin Infect Dis. 2004;39(2):165-169.

30. Atlas SJ, Benzer TI, Borowsky LH, et al. Safely increasing the proportion of patients with community-acquired pneumonia treated as outpatients: an interventional trial. Arch Intern Med. 1998;158:1350-1356.

31. Feagan BG, Marrie TJ, Lau CY, et al. A critical pathway for treatment of community-acquired pneumonia. JAMA. 2000; 283:2654-2655.

32. Carratala J, Fernandez-Sabe N, Ortega L, et al. Outpatient care compared with hospitalization for community-acquired pneumonia. A randomized control trial in low-risk patients. Ann Intern Med. 2005;142:165-172.

33. Ramsdell J, Narsavage GL, Fink JB. Management of community-acquired pneumonia in the home. Chest. 2005;127: 1752-1763.

34. Gleason PP, Meehan TP, Fine JM, Galusha DH, Fine MJ. Associations between initial antimicrobial therapy and medical outcomes for hospitalized elderly patients with pneumonia. Arch Intern Med. 1999;159:2562-2572.

35. Houck PM, Bratzler DW, Nsa W, Ma A, Bartlett JG. Timing of antibiotic administration and outcomes for Medicare patients hospitalized with community-acquired pneumonia. Arch Intern Med. 2004;164:637-644.

36. Whitney CG, Farley MM, Hadler J, et al. Increasing prevalence of multidrug-resistant Streptococcus pneumoniae in the United States. N Engl J Med. 2000;343:1917-1924.

37. Pottumarthy S, Fritsche TR, Sader HS, et al. Susceptibility patterns of Streptococcus pneumoniae isolates in North America (2002-2003): contemporary in vitro activities of amoxicillin/clavulanate and 15 other antimicrobial agents. Int J Antimicrob Agents. 2005;25(4):282-289.

38. Doern GV, Richter SS, Miller A, et al. Antimicrobial resistance among Streptococcus pneumoniae in the United States: have we begun to turn the corner on resistance to certain antimicrobial classes? Clin Infect Dis. 2005;41(2):139148. 
39. Ewig S, Ruiz M, Torres A, et al. Pneumonia acquired in the community through drug-resistant Streptococcus pneumoniae. Am J Respir Crit Care. 1999;159:1835-1842.

40. Aspa J, Rajas O, Rodriguez de Castro F, et al. Drug-resistant pneumococcal pneumonia: clinical relevance and related factors. Clin Infect Dis. 2004;38:787-798.

41. Feikin DR, Schuchat A, Kolczak M, et al. Mortality from invasive pneumococcal pneumonia in the era of antibiotic resistance, 1995-1997. Am J Public Health. 2000;90(2):223-9.

42. Garcia Vazquez E, Mensa J, Martinez JA, et al. Lower mortality among patients with community-acquired pneumonia treated with a macrolide plus a beta-lactam agent versus a beta-lactam alone. Eur J Clin Microbiol Infect Dis 2005;24: 190-195.

43. Brown RB, Iannini P, Gross P, Kunkel M. Impact of initial antibiotic choice on clinical outcomes in community-acquired pneumonia: analysis of a hospital claims-made database. Chest. 2003;123:1503-1511.

44. Dudas V, Hopefl A, Jacobs R, Guglielmo BJ. Antimicrobial selection for hospitalized patients with presumed community-acquired pneumonia: a survey of nonteaching US community hospitals. Ann Pharmacother 2000;34:446-452.

45. Flanders SA, Dudas V, Kerr K, McCullough CE, Gonzales R. J Hosp Med. 2006;1:7-12.

46. Amsden GW. Anti-inflammatory effects of macrolides-an underappreciated benefit in the treatment of communityacquired respiratory tract infections and chronic inflammatory pulmonary conditions? J Antimicrob Chemother. 2005; 55:10-21.

47. Martinez JA, Horcajada JP, Almela M, et al. Addition of a macrolide to a beta-lactam based empirical antibiotic regimen is associated with lover in-hospital mortality for patients with bacteremic pneumococcal pneumonia. Clin Infect Dis. 2003;36:389-395.

48. Baddour LM, Yu VL, Klugman KP, et al. Combination antibiotic therapy lowers mortality among severely ill patients with pneumococcal bacteremia. Am J Respir Crit Care Med. 2004; 170:440-444.

49. Shefet D, Robenshtok E, Paul M, Leibovici L. Empiric antibiotic coverage of atypical pathogens for community-acquired pneumonia in hospitalized adults. Cochrane Database Syst Rev. 2005;2:CD004418.pub2.

50. Mills GD, Oehley MR, Arrol B. Effectiveness of $\beta$ lactam antibiotics compared with antibiotics active against atypical pathogens in non-severe community-acquired pneumonia: meta-analysis. Br Med J. 2005;330:456.

51. Shefet D, Robenshtok Y, Paul M, Leibovici L. Empirical atypical coverage for inpatients with community-acquired pneumonia. Arch Intern Med. 2005;165:1992-2000.

52. Neuhauser MM, Weinstein RA, Rydman R, et al. Antibiotic resistance among gram-negative bacilli in US intensive care units: implications for fluoroquinolone use. JAMA. 2003;289: 885-888.

53. Nseir S, Di Pompeo C, Soubrier S, et al. First-generation fluoroquinolone use and subsequent emergence of multiple drug-resistant bacteria in the intensive care unit. Crit Care Med. 2005;33(2):283-289.

54. Apisarnthanarak A, Mundy LM. Etiology of community-acquired pneumonia. Clin Chest Med. 2005;26:47-55.

55. File TM. Community-associated methicillin-resistant Staph- ylococcus aureus: not only a cause of skin infections, also a new cause of pneumonia. Curr Opin Infect Dis. 2005;18:123124.

56. Francis JS, Doherty MC, Lopatin U, et al. Severe communityonset pneumonia in healthy adults caused by methicillinresistant Staphylococcus aureus carrying the Panton-Valentine leukocidin genes. Clin Infect Dis. 2005;40(1):100-107.

57. Frazee BW, Salz TO, Lambert L, Perdreau-Remington F. Fatal community-associated methicillin-resistant Staphylococcus aureus pneumonia in an immunocompetent young adult. Ann Emerg Med. 2005;46:401-404.

58. Marik PE. Aspiration pneumonitis and aspiration pneumonia. $N$ Engl J Med. 2001;344:665-671.

59. Friedman ND, Kaye KS, Stout JE, et al. Health care-associated bloodstream infections in adults: a reason to change the accepted definition of community-acquired infections. Ann Intern Med. 2002;137:791-797.

60. American Thoracic Society and the Infectious Diseases Society of America. Guidelines for the management of adults with hospital-acquired, ventilator-acquired, and healthcareassociated pneumonia. Am J Respir Crit Care Med. 2005;171: 388-416.

61. Chang NN, Murray CK, Hourck PM, et al. Blood culture and susceptibility results and allergy history do not influence fluoroquinolone use in the treatment of community-acquired pneumonia. Pharmacotherapy. 2005;25(1):59-66.

62. Halm EA, Fine MJ, Marrie TJ, et al. Time to clinical stability in patients hospitalized with community-acquired pneumonia: implications for practice guidelines. JAMA. 1998;279: 1452-1457.

63. Dunbar LM, Wunderink RG, Habib MP, et al. High-dose, short-course levofloxacin for community-acquired pneumonia: a new treatment paradigm. Clin Infect Dis. 2003;37: 752-760.

64. Roson B, Carratala J, Fernandez-Sabe N, et al. Causes and factors associated with early failure in hospitalized patients with community-acquired pneumonia. Arch Intern Med. 2004;164:502-508.

65. Halm EA, Fine MJ, Kapoor WN, et al. Instability on hospital discharge and the risk of adverse outcomes in patients with pneumonia. Arch Intern Med. 2002;162:1278-1284.

66. El Solh AA, Aquilina AT, Gunen H, Ramadan F. Radiographic resolution of community-acquired bacterial pneumonia in the elderly. J Am Geriatr Soc. 2004;52(2):224-229.

67. Dear KB G, Andrews RR, Holden J, Tatham DP. Vaccines for preventing pneumococcal infection in adults. Cochrane Database Syst Rev. 2003;4:CD000422.

68. Gross PA, Hermogenes AW, Sacks HS, Lau J, Levandowski RA. The efficacy of influenza vaccine in elderly persons: a meta-analysis and review of the literature. Ann Intern Med. 1995;123:518-527.

69. Nichol KL, Nordin J, Mullooly J, et al. Influenza vaccination and reduction in hospitalizations for cardiac disease and stroke among the elderly. $N$ Engl J Med. 2003;348:1322-1332.

70. Almirall J, Gonzalez CA, Balanzo X, Bolibar I. Proportion of community-acquired pneumonia cases attributable to tobacco smoking. Chest. 1999;116:375-379.

71. Laheij RJF, Sturkenboon MC, Hassing R, et al. Risk of community-acquired pneumonia and use of gastric acid-suppressive drugs. JAMA. 2004;292:1955-1960. 